\title{
Analyzing Spiral Galaxies Observed in Near-Infrared
}

\author{
Preben Grosbøl \\ European Southern Observatory \\ Karl-Schwarzschild-Str. 2, D-85748 Garching, Germany
}

\begin{abstract}
A sample of 54 spiral galaxies was observed in the near-infrared $K^{\prime}$ band and used to analyze perturbations in galactic disks. Although it is desirable to perform reductions and analysis as automatic as possible, some steps require manual intervention. These are discussed together with several techniques useful for analysis of spiral galaxies.
\end{abstract}

\section{INTRODUCTION}

With improved detectors for near-infrared (NIR) wavelengths, it is now feasible to make deep surface photometry in these bands. Observations in the $2.1 \mu \mathrm{K}^{\prime}$ band provide an option to study the mass distribution of the old stellar population in galaxies without strong population effects or dust attenuation (Rix \& Rieke 1993). Although other components (e.g. dark matter halos) also are very important for the dynamics, vital information on perturbations in disks of galaxies can be obtained from NIR surface brightness maps (e.g. location, strength and shape of spiral/bar modes).

Whereas the analysis of individual galaxies may yield interesting results, only the study of larger samples will indicate the general properties. One challenge for such studies is to automatize as many steps as possible without introducing an un-acceptable large amount of gross errors. This paper $^{1}$ considers the analysis of a medium size sample of 50-100 spiral galaxies observed in the $\mathrm{K}^{\prime}$ band. The objective was to model the mass distribution of the galaxies and study perturbations in their disks. The steps which required some manual intervention are considered and some useful techniques are discussed.

\section{OBJECTIVES AND DATA}

The main aim of the current study was to measure the detailed amplitude and shape of bar/spiral perturbations in the disks of spiral galaxies and thereby provide a database which can be used to test theoretical model of such phenomena e.g. the density wave theory by Lin and Shu (1964). For this purpose, 54 spiral galaxies were observed in $\mathrm{K}^{\prime}$ with SOFI at the $3.5 \mathrm{NTT}$, La Silla. They were selected to have a wide range of morphological types, inclination angles $i<65^{\circ}$ to ensure that spiral structure in their disks could be seen, and with diameters small enough to be observed with one pointing of the $5^{\prime}$ SOFI field.

\section{HOW AUTOMATED CAN THE ANALYSIS BE?}

Although spiral galaxies have a much smoother appearance in NIR than in visible bands, they still are not exactly fitted by a simple analytical model. This makes it difficult to apply automatic procedures for the analysis. The main steps which need some manual attention are list below:

Rejection of raw frames: Even though NIR detectors have improved significantly during the last years, they do not reach the stability of CCD's in the visual. Whereas frames with gross error can rejected automatically, one frequently sees images with more subtle patterns which

\footnotetext{
${ }^{1}$ Based on observations collected at the European Southern Observatory, La Silla, Chile; programs: ESO 63.N-0343, 65.N-0287, 66.N-0257.
} 


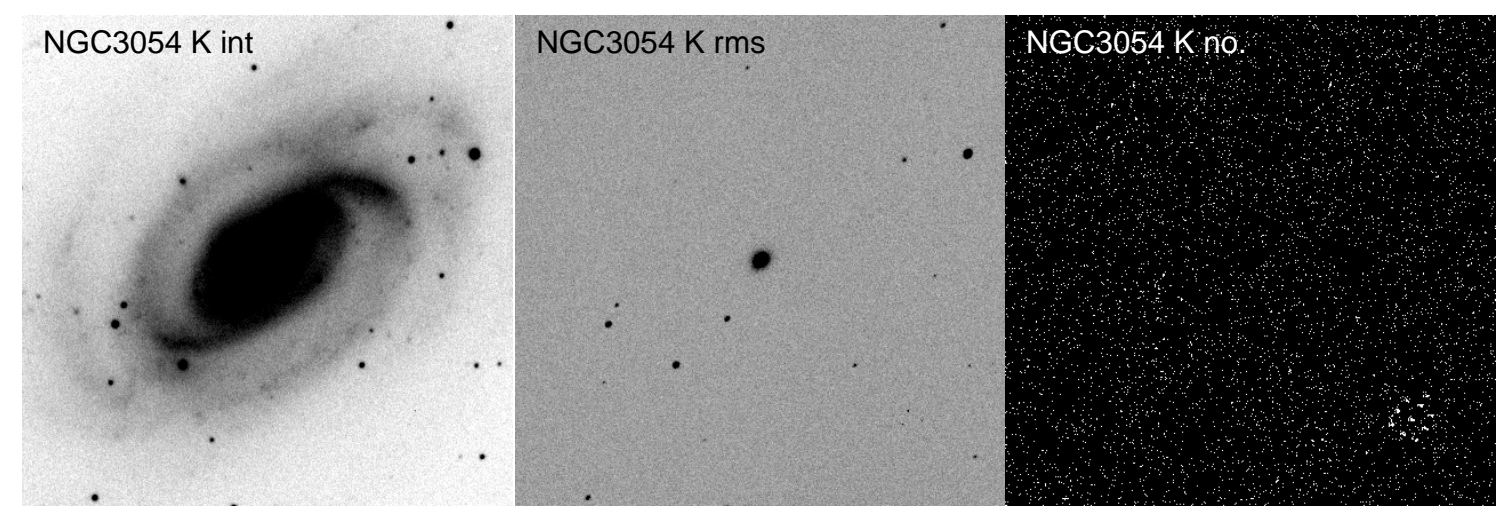

FIGURE 1: Maps of NGC 3054 observed in $\mathrm{K}^{\prime}$ band in negative representation. The maps are the average of the 30 frame stack, rms of the average and number of frames used for the average, respectively.

obviously are due to detector problems but only can be identified and rejected by visual inspection.

Identification of foreground stars: Point sources in the field can be found using standard search algorithms. Close to extended sources such as galaxies, the detection becomes more difficult and many sources may be missed. Manual identification is the only safe way to locate foreground stars which may disturb the analysis of a galaxy and to mask off the corresponding areas.

Estimates of projection parameters: Especially strong open spiral patterns may bias the determination of position and inclination angles of a galaxy. One may use several different methods with different biases to find such difficult cases. In the end, visual inspection is needed to understand the reason for the differences and select the better estimate.

Measurement of extent of bar and main spiral: The phase and amplitude of spiral perturbations can be measured by using Fourier techniques. Bars can be defined as bisymmetric perturbations with constant phase. In principle it should be easy to locate the end of a bar and the starting of the spiral pattern as the radius where the phase of the bisymmetric perturbation change from being constant to having a well define slope as function of radius. Real spiral galaxies often have a complex structure in the interface region between bar and spiral which makes it almost impossible to rely on automatic method. The extent of the main spiral pattern is also hard to determine by automated procedures as multiple spiral mode may be present and its termination is gradual.

\section{USEFUL TECHNIQUES}

During the reductions and analysis of this sample of spiral galaxies, several techniques and algorithms turned out to give more stable results than those of standard procedures. Three of these are discussed below.

\subsection{Stacking of frames}

The high sky background in NIR makes it necessary to break up exposures in to many short ones to avoid saturation of the detectors. Depending on the actual conditions and wavelength, the observation of a field may consist of a stack of 10-30 frames with slightly different pointings. This makes it possible to compare the individual pixel values in the stack after aligning it and thereby reject outliers (e.g. bad pixels or cosmic ray events) and estimate the actual uncertainty.

Median filters or $\kappa \sigma$-clipping is often used for this purpose. Although they work well in most cases, high gradient regions (e.g. bright stars or centers of galaxies) may produce a double peaked distribution of pixel values due to small misalignments which can cause these methods to be unstable. 


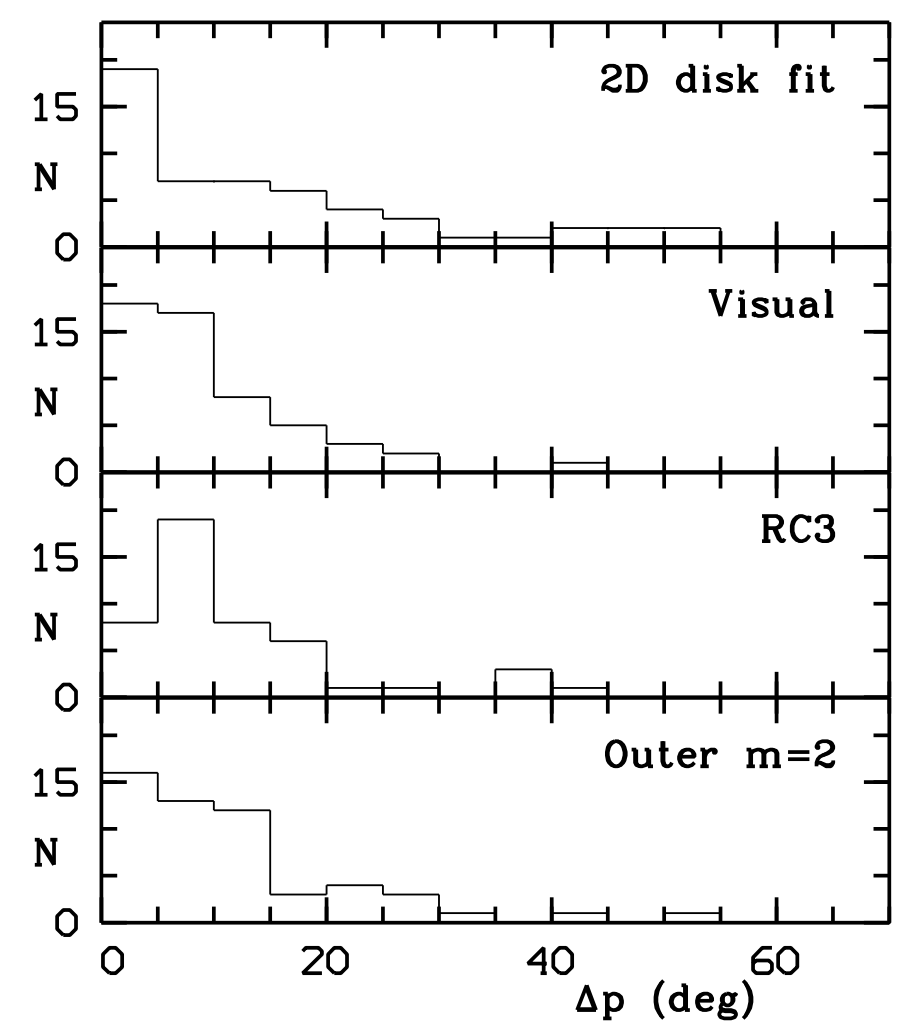

FIGURE 2: Histograms of the angular distances between the normals to the plane of the disk estimated by minimizing the bar components in the disk and other determinations such as a) 2D fit of an exponential disk, b) visual or interactive estimate, c) RC3 projection parameters, and d) minimizing the bisymmetric $\mathrm{m}=2$ variation in the outer parts of the disk.

An alternative algorithm is to rank the stack values and compute its variance and uncertainty of this estimate. By removing extreme values, one can check if the exclusion gave raise to a reduction of the variance at a given level of significance. Pixel which significantly increase the variance can then be rejected. This method is more stable for multi-peak distributions.

As an example, the result of reducing a stack of 30 frames of the galaxy NGC 3054 observed in $\mathrm{K}^{\prime}$ is shown in Fig. 1 where both average, rms and number of frames used are displayed. The rms is only higher at strong sources due to photon noise while most of the image has the same level given by the high background. Even in high gradient regions close to strongest sources one can not seen any increase in number of rejected pixels while areas of bad pixels still clearly are rejected correctly.

\subsection{Estimating projection parameters}

It is non-trivial to determine the sky projection parameters for spiral galaxies from surface brightness maps due to spiral structure and possible warps in their disks. Several standard methods are available such as measuring the axes ratio, fitting ellipses to the outer isophotes, fitting a galaxy model containing bugle and exponential disk to the galaxies, and analyzing the radial phase variation of spiral arms (Danver 1942). Most of these algorithms work well for galaxies with weak, tight spiral arms but may yield significant errors for strong, open spirals. Several of them are based on the outer regions of the disk which could be warped.

Another option is compute the 2D Fourier transform of the disk in polar coordinates and minimize the amplitude of the constant phase bisymmetric component which mainly is produced by projection effects. An advantage of this algorithm is that it can be applied to the inner parts of the disk which has a higher surface brightness since it reduces the influence of the spiral pattern. 


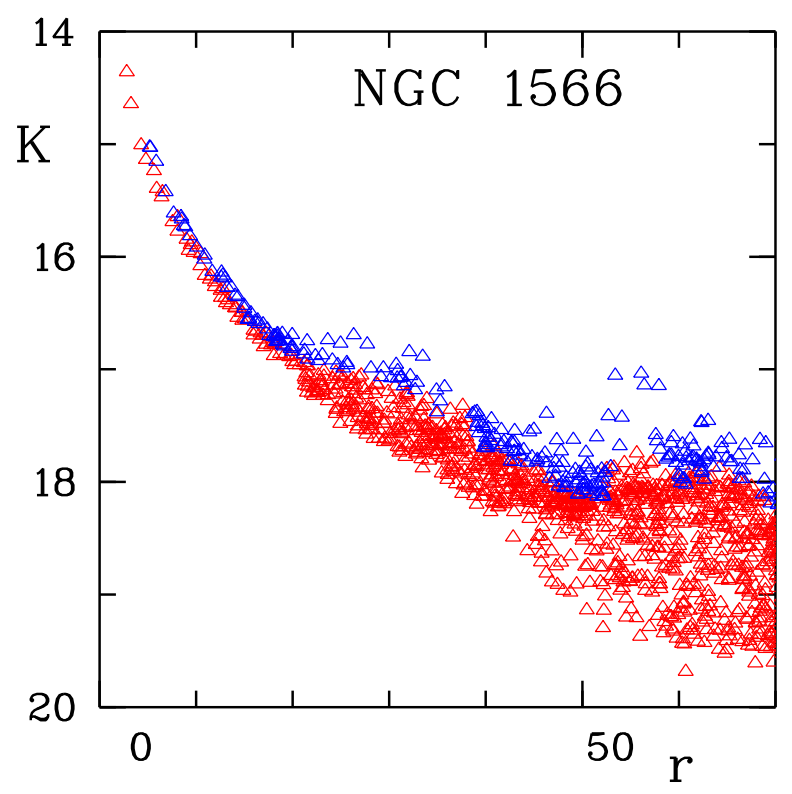

FIGURE 3: Distribution of surface brightness of resolution elements of $1^{\prime \prime}$ in NGC 1566 observed in $\mathrm{K}^{\prime}$ as function of radius. Pixels defined to be in the inter-arm regions are shown in red while arm regions are indicated by blue.

The main problem is that it must be used in regions outside any real bar component in the galaxy as this would bias the estimate.

A comparison of this and other methods is shown in Fig. 2 where the distributions of angular distances between normals to the disks derived by the different methods are given. Although there is a tail of gross errors, it is consistent with both 2D fits and visual estimates for well behaved galaxies.

\subsection{Deriving properties for the disks}

To analyze perturbations in the disks of spiral galaxies, one needs to estimate the axisymmetric distribution of visual material in the disk. This is typically done by fitting an exponential disk to the surface brightness of the old stellar disk population as measured in the $\mathrm{K}^{\prime}$ band (Kent 1985). Although absorption by dust can be ignored, population effects still exist as indicated by the many bright knots often seen along spiral arms.

The influence of young objects can largely be avoided by only using the inter-arm regions to estimate the disk properties. These regions can be defined as all pixels with a value less than the mean of the minimum and maximum intensities at a given radius in the plane of the disk. This definition includes more pixels than if the average radial intensity was used but avoids the bright knots associated to young objects.

The distribution of pixel values in a $K^{\prime}$ map of NGC 1566 is shown in Fig. 3 as function of the radius. It is clear that only a relative small amount of pixels (blue) are defined to be associated to arms. These pixels have a longer exponential scale length than the inter-arm pixels and can therefore bias the fits. Differences of up to $10 \%$ are seen when comparing estimates based on inter-arm regions and the full disk (Grosbøl et al. 2004). Although the disk properties derived form the inter-arm regions have a slightly underestimate central intensity, the scale length better represents that of the old disk population. 


\section{CONCLUSIONS}

The reduction and analysis of NIR images of spiral galaxies can largely be done automatically but needs at several stages manual supervision. The estimation of projection parameters for strong, open spiral needs special attention while the extent of bars and main spiral arms only can be determined safely by visual inspection.

It is important to use stable algorithms when attempting to perform automated processing. Improvements can be made in areas such as stacking of frames and estimation of projection parameters.

\section{REFERENCES}

[1] Danver, C.-G. 1942, Ann. Obs. Lund, 10

[2] Kent, S. M. 1985, ApJS, 59, 115

[3] Lin, C. C. \& Shu, F. H. 1964, ApJ, 140, 646

[4] Grosbøl, P., Patsis, P. A. \& Pompie, E. 2004, A\&A, in press

[5] Rix, H.-W. \& Rieke, M. J. 1993, ApJ, 418, 123 